\title{
Tasa relativa de crecimiento en plántulas de dos poblaciones de Magnolia pugana (Magnoliaceae) en distintos niveles de luz y fertilidad del suelo
}

\author{
Karla Denisse García Castro ${ }^{1}$, Rosa de Lourdes Romo-Campos ${ }^{2 *}$, César Jacobo Pereira ${ }^{1}$ \\ \& Raúl Gómez-Rubio ${ }^{1}$ \\ 1. Egresado de la Licenciatura en Biología, Departamento de Ciencias Ambientales, Centro Universitario de Ciencias \\ Biológicas y Agropecuarias, Universidad de Guadalajara. México; karla-c17@hotmail.com, \\ bio.cesarjacobo@gmail.com, raulpc89@hotmail.com \\ 2. Departamento de Ciencias Ambientales, Centro Universitario de Ciencias Biológicas y Agropecuarias. Universidad \\ de Guadalajara. Camino Ramón Padilla Sánchez 2100, Nextipac, 44600 Zapopan, Jalisco, México; \\ rlrc.cucba@gmail.com
}

Recibido 25-X-2017. Corregido 18-I-2018. Aceptado 22-II-2018.

\begin{abstract}
Relative growth rate in Magnolia pugana (Magnoliaceae) seedlings from two populations at different light levels and soil fertility. The study of ecophysiology of endangered species is key to the success of conservation and ecological restoration programs. The objective of this work was to know the effect of light, soil fertility and seed origin on the growth of Magnolia pugana seedlings. The relative growth rate (RGR) and its components (Net Assimilation Rate: NAR and Leaf Area Ratio: LAR), as well as the root-shoot ratio (R/S) were estimated. Seedlings were obtained from seeds collected in two localities in Zapopan, Jalisco, Mexico, the first is a wild population in San Nicolás (SN) and the second is a plantation located in the gardens of the Centro Universitario de Ciencias Biológicas y Agropecuarias (CUCBA). The experiment was conducted under greenhouse conditions. In September 2015, 96 seedlings of approximately three months old were subjected to the following treatments in a factorial experiment $(2 \times 2 \times 2)$ : light level (high $=1120 \mu \mathrm{mol} \mathrm{m} \mathrm{m}^{-2} \mathrm{~s}^{-1}$ and low $=$ $136.3 \mu \mathrm{mol} \mathrm{m} \mathrm{m}^{-2} \mathrm{~s}^{-1}$ ), seedling origin ( $\mathrm{SN}$ and CUCBA) and soil fertility (high fertility $=\mathrm{SN}$ soil, low fertility = CUCBA soil) with 12 replicates for each combination of factors and levels. Growth was estimated in two harvests: the first harvest after 30 days of starting the experiment and the second at 60 days. Light and soil fertility levels, as well as seedling origin influenced growth through physiological and morphological adjustments. In general, Magnolia pugana showed higher RGR and NAR in high light, while LAR decreased. Soil fertility and seedling origin did not affect RGR or its components. However, these were affected by the light level, seedlings from SN grew more in high light. Stem height varied due to soil fertility and seedling origin, in low fertility soil (CUCBA) the seedlings of SN were $35 \%$ higher than those of CUCBA. Low fertility soil under high light caused greater investment in seedling root biomass. The physiological component (NAR) was the major determinant of intraspecific variation in RGR. SN seedlings showed greater phenotypic plasticity due to coming from a wild population, which possibly has higher genetic variation than the cultivated population from CUCBA. The results suggest that Magnolia pugana is a species capable of adapting to diverse environments due to its phenotypic plasticity in response to different light and soil fertility levels. Rev. Biol. Trop. 66(2): 622-633. Epub 2018 June 01.
\end{abstract}

Key words: Magnolia pugana; relative growth rate; leaf area ratio; net assimilation rate; growth analysis; endangered species.

La reintroducción de especies amenazadas a su hábitat natural, es una estrategia de conservación ex situ, que proporciona un respaldo de seguridad frente a la extinción de las poblaciones (Sharrock, 2012). El éxito para la restauración ecológica de poblaciones en riesgo depende del conocimiento del crecimiento y desempeño fisiológico de las plantas durante su estadio más vulnerable, la fase de plántula (Restrepo \& Cardona, 2011). 
El crecimiento y los patrones de asignación de biomasa en las plántulas, es afectado tanto por factores intrínsecos de la especie tales como: el tamaño de su semilla (determinado por el genotipo de la planta), así como por las condiciones ambientales durante su desarrollo (Fenner \& Kitajima, 1999; Herrera, Alizaga, Guevara, \& Jiménez, 2006). Dentro de estas últimas se encuentran la intensidad lumínica que llega a nivel del sotobosque y la disponibilidad de nutrientes minerales, los cuales son factores ambientales relevantes en el crecimiento, supervivencia y distribución de las especies de plantas (Lambers, Chapin, \& Pons, 1998; Azcon-Bieto \& Talon, 2008). Una de las medidas principales para el análisis del crecimiento en plantas es la tasa relativa de crecimiento (TRC), definida como la ganancia de biomasa por unidad de biomasa y tiempo (Hoffmann \& Poorter, 2002; Alameda \& Villar, 2009).

Las plantas que crecen adaptadas a la sombra responden a esta condición asignando mayor biomasa a los órganos fotosintéticos para aumentar el área de exposición y recibir más luz (Pearcy, Valladares, Wright, \& de Paulis, 2004; Villar et al., 2004), con esto incrementa la proporción del área foliar por unidad de biomasa total de la planta (cociente del área foliar, CAF) (Valladares, Aranda, \& Sánchez-Gómez, 2008); además, muestran menor tamaño de la raíz (Villar et al., 2004). Por el contrario, en ambientes de alta disponibilidad de luz, las plantas generalmente presentan tasas de asimilación neta (TAN) más altas y mayor inversión de biomasa para las raíces, debido a que el crecimiento es estimulado, lo cual implica una mayor absorción de agua y nutrientes (Poorter, 2001). También la disponibilidad de nutrientes es clave en las diferencias morfo-fisiológicas durante el crecimiento de las plantas (Poorter \& Nagel, 2000). En suelos con baja fertilidad el crecimiento está limitado, por lo que éstas asignan mayor biomasa a la raíz para captar más agua y nutrientes y el resultado es un mayor cociente raíz/vástago $(\mathrm{C}: \mathrm{R} / \mathrm{V})$ (Huante, Rincon, \& Acosta, 1995; Romo-Campos, Flores-Flores, Flores, \& Álvarez-Fuentes, 2013).
La familia Magnoliaceae, es uno de los grupos más primitivos de las angiospermas (Cicuzza, Newton, \& Oldfield, 2007). Actualmente se encuentra integrada por árboles y arbustos de hoja perenne o caduca, con únicamente dos géneros, Liriodendron L. y Magnolia L. (Glimn-Lacy \& Kaufman, 2006). Esta familia está constituida por ca. 330 especies; distribuidas irregularmente en las zonas tropicales, subtropicales y templadas del sureste de Asia, sureste de Norte América, América central y norte de Sudamérica y más del $50 \%$ de sus especies están en la Lista Roja de la UICN con alguna categoría de amenaza de extinción. (Vázquez-García et al., 2014; Rivers, Martínez-Salas, \& Samain, 2016).

Magnolia pugana (H. H. Iltis y A. Vázquez) A. Vázquez \& Carvajal, es una especie endémica del occidente de México, es localmente llamada Almacasusco, forma parte de los bosques de galería presentes en la región de barrancas y cañadas del Río Santiago en el centro-norte del estado de Jalisco y sur de Zacatecas (Vázquez-García, 1994; Vázquez-García, Carbajal, \& Hernández, 2002). La fragmentación de su hábitat por la ganadería extensiva, el crecimiento urbano, los incendios forestales y el aprovechamiento forestal para autoconsumo, han provocado el descenso de sus poblaciones. Actualmente está catalogada como especie en peligro crítico de extinción (Rivers et al., 2016) debido a su distribución restringida y al tamaño pequeño de sus poblaciones, factores como la endogamia y la deriva genética podrían afectar sus poblaciones y favorecer escenarios de extinción (Vázquez-García et al., 2002; Carranza, 2014). La restauración de poblaciones de $M$. pugana requiere atención urgente, por lo que es prioritario conocer su respuesta en edades tempranas a factores como la luz y tipo de suelo, lo que podría favorecer la reintroducción de individuos a su hábitat natural. El objetivo de esta investigación fue evaluar en plántulas de Magnolia pugana los efectos de la luz, fertilidad del suelo y procedencia de las semillas en la tasa relativa de crecimiento y sus componentes con el fin de contribuir al conocimiento de su ecofisiología. 


\section{MATERIALES Y MÉTODOS}

Área de estudio: La zona de estudio comprende dos localidades en el municipio de Zapopan, Jalisco, México. La primera es una plantación en el Centro Universitario de Ciencias Biológicas y Agropecuarias (CUCBA) con coordenadas $20^{\circ} 44^{\prime} 53.6^{\prime \prime}$ - 103'30'52.2” W, a una altitud de $1659 \mathrm{msnm}$, la cual contiene 25 individuos adultos de Magnolia pugana que fueron plantados como brinzales en 1985, provenientes de semillas recolectadas de una población silvestre ubicada en las inmediaciones del poblado de San Lorenzo $(8 \mathrm{~km}$ al Oeste del pueblo de Santa Lucia, Zapopan, Jalisco) (Jacobo-Pereira，Romo-Campos，\& Flores, 2016). Los suelos predominantes son de tipo Regosol eútrico, los cuales se caracterizan por su pobre contenido en materia orgánica y una textura arenosa (Ibarra, Ruiz, González, \& Flores, 2007). El clima es semicálido subhúmedo con lluvias en verano, la temperatura media anual es de $20.6{ }^{\circ} \mathrm{C}$, la temperatura mínima y máxima promedio corresponden a los meses de enero y mayo con $8.2{ }^{\circ} \mathrm{C}$ y $33.4{ }^{\circ} \mathrm{C}$ respectivamente. La precipitación promedio anual es de $999.3 \mathrm{~mm}$, estos datos fueron tomados de la estación climática de la Comisión Nacional del Agua de Zapopan, Jalisco (Ruiz, Flores, Regalado, \& Ramírez, 2012).

La segunda localidad (San Nicolás) es una población silvestre localizada en el arroyo $\mathrm{La}$ Virgen, a $300 \mathrm{~m}$ aproximadamente del poblado de San Nicolás (SN), Zapopan, Jalisco, con coordenadas $20^{\circ} 48^{\prime} 53.4^{\prime \prime}$ - 103'34'48.9" W a una altitud de $1445 \mathrm{msnm}$. Los suelos son Regosol eútrico, Cambisol eútrico y Feozem háplico que se caracterizan por ser someros con profundidad menor a $10 \mathrm{~cm}$ y de textura media (SPP, 1981). El clima de este sitio es similar al anterior debido la cercanía de ambas localidades (aproximadamente $20 \mathrm{~km}$ entre sí). El bosque de galería es el hábitat natural de Magnolia pugana, las principales especies que la acompañan son: Aphananthe monoica (Hemsl.) J.-F. Leroy, Ficus insipida Willd., Litsea glaucescens Kunth, Lysiloma acapulcense (Kunth) Benth, Oreopanax peltatus Linden ex Regel,
Piper hispidum Sw., P. jaliscanum S. Watson, Prunus ferruginea Steud., Salix humboldltiana Willd., Taxodium mucronatum Ten. y Xanthosoma robustum Schott (Acevedo-Rosas, Hernández-Galaviz, \& Cházaro-Basáñez, 2008).

Recolecta de semillas y crecimiento de plántulas en invernadero: A finales de abril 2015 se recolectaron semillas procedentes de frutos maduros de por lo menos 10 individuos adultos por localidad (CUCBA, San Nicolás). Los polifolículos que se encontraban a punto de madurar fueron envueltos en papel estraza para acelerar su maduración. Posteriormente, las semillas se extrajeron del fruto manualmente, y sin retirar el arilo se lavaron en una solución de hipoclorito de sodio $(\mathrm{NaClO})$ al $3 \%$ para eliminar la contaminación por hongos (GarcíaRamírez, Freire-Seijo, Tejeda, \& Reyes, 2007). A su vez, estas se conservaron a $4-5^{\circ} \mathrm{C}$, por un periodo no mayor de 30 días para evitar la deshidratación (Saldaña, Zuloaga, \& Jardel, 2001).

El experimento se llevó a cabo en un invernadero ubicado en Centro Universitario de Ciencias Biológicas y Agropecuarias de la Universidad de Guadalajara (2044'53.6" $\mathrm{N}$ - 103 30'52.2” W). Para lograr un mayor porcentaje de germinación el arilo de las semillas fue removido (Jacobo-Pereira et al., 2016). Seiscientas semillas fueron sembradas manualmente (300 por localidad) a una profundidad de $1.5 \mathrm{~cm}$, en 12 charolas de plástico de 50 cavidades de 27 x $54 \mathrm{~cm}$ y $4 \mathrm{~cm}$ de profundidad, cada una. Se utilizó como sustrato suelo de encino y jal (roca volcánica ácida de carácter vítreo o pumicita), (SEMARNAT, 2000) en proporción 2:1. Las charolas se regaron diariamente a capacidad de campo hasta su germinación, cuando el hipocótilo quedó expuesto (Bewley \& Black, 1994).

El trasplante de las plántulas se llevó a cabo en septiembre 2015. Las plántulas se trasplantaron individualmente en bolsas de plástico biodegradables de $25 \times 18 \mathrm{~cm}$ con 2 $\mathrm{kg}$ de suelo que procede de los sitios de estudio (suelo de CUCBA y suelo de SN), y fueron regadas cada tercer día a capacidad de campo. Para conocer las diferencias entre los suelos 
CUADRO 1

Promedio de los atributos del suelo por localidad $(n=3)$

TABLE 1

Averages of soil properties by locality $(n=3)$

\begin{tabular}{lccc} 
& & San Nicolás & CUCBA \\
Pextura & Arena & $78.5 \pm 3.4$ & $78.3 \pm 1.7$ \\
& Limo & $17.9 \pm 2.4$ & $9.9 \pm 0.7$ \\
& Arcilla & $3.5 \pm 0.3$ & $11.7 \pm 1.0$ \\
Materia orgánica \% & - & $31.9 \pm 0.3$ & $8.4 \pm 0.7$ \\
$\mathrm{pH}$ & - & $6.3 \pm 0.07$ & $4.5 \pm 0.09$ \\
Capacidad de intercambio catiónico & - & $52.3 \pm 2.4$ & $25.7 \pm 0.07$ \\
Cationes & $\mathrm{Ca} \mathrm{meq/100} \mathrm{g}$ & $23.9 \pm 0.8$ & $3.6 \pm 0.04$ \\
Intercambiables & $\mathrm{Mg} \mathrm{meq} / 100 \mathrm{~g}$ & $10 \pm 0.15$ & $2.5 \pm 0.07$ \\
& $\mathrm{Na} \mathrm{meq} / 100 \mathrm{~g}$ & $0.88 \pm 0.001$ & $0.15 \pm 0.02$ \\
& $\mathrm{~K} \mathrm{meq} / 100 \mathrm{~g}$ & $3.3 \pm 0.3$ & $4.3 \pm 0.6$ \\
Nutrientes & $\mathrm{P} \mathrm{ppm}$ & $66 \pm 19.5$ & $4.5 \pm 0.29$ \\
& $\mathrm{~K} \mathrm{ppm}$ & $1081 \pm 100$ & $1006 \pm 35.7$ \\
& $\mathrm{Ca} \mathrm{ppm}$ & $4140 \pm 344$ & $607 \pm 4$ \\
& $\mathrm{Mg} \mathrm{ppm}$ & $867 \pm 106$ & $268 \pm 8.7$ \\
\hline
\end{tabular}

Promedio \pm error estándar.

se les realizó un análisis de las características físico-químicas en el Laboratorio de Suelos del CUCBA (Cuadro 1). Para la evaluación de la tasa de crecimiento y sus componentes, se seleccionaron un total de 96 plántulas (48 para CUCBA y 48 para SN) de aproximadamente tres meses de edad y tamaño similar.

El diseño del experimento fue completamente al azar, factorial $2 \times 2 \times 2$ con dos niveles por cada factor: fertilidad dada por la procedencia del suelo (baja: CUCBA y alta: suelo de $\mathrm{SN}$ ), procedencia de las plántulas (plántulas del CUCBA y plántulas de SN), y luz (alta: $100 \%$ y baja: $10 \%$ de luz, inducida mediante una malla de sombra), con 12 repeticiones por cada combinación de tratamientos. Las macetas dentro de cada tratamiento fueron ubicadas al azar. Una vez establecidos los tratamientos se registró la temperatura y humedad relativa del ambiente con un dispositivo electrónico, HOBO H08-004-02 (Onset Computer Corporation, Massachusetts, USA), así como la densidad de flujo de fotones fotosintéticos
(DFFF) mediante un Quantum LI-COR LI-250 hasta el término del experimento.

Las condiciones en el invernadero a las que estuvieron sometidos los tratamientos fueron las siguientes: en luz alta, la temperatura promedio fue de $36.57{ }^{\circ} \mathrm{C}$ y la humedad relativa promedio de $56.15 \%$. Mientras que, en luz baja la temperatura promedio fue de $18{ }^{\circ} \mathrm{C}$ y la humedad relativa promedio el $75.6 \%$. La densidad de flujo de fotones fotosintéticos (DFFF) máxima registrada para el tratamiento de luz alta fue de $1120.3 \mu \mathrm{mol} \mathrm{m} \mathrm{m}^{-2} \mathrm{~s}^{-1} \mathrm{y}$ en luz baja de $136.3 \mu \mathrm{mol} \mathrm{m}^{-2} \mathrm{~s}^{-1}$.

Variables de respuesta: el crecimiento se evaluó a través de la tasa relativa de crecimiento (TRC). Para estimarla se realizaron dos cosechas (seis plántulas por cosecha): la primera a 30 días después de establecido el experimento y la segunda a los 60 días. El día anterior a la segunda cosecha se registró para cada plántula la altura final del tallo y el número de hojas con tres repeticiones por tratamiento. El área foliar se obtuvo mediante la digitalización de 
imágenes en un escáner marca HP Officejet Pro 8600 que posteriormente fueron analizadas con el software Sigma Scan/image (Jandel Scientific, 1993). Para obtener el peso seco se separaron las hojas, raíces y tallos y se introdujeron en un horno a $70{ }^{\circ} \mathrm{C}$ hasta llegar a peso constante.

La TRC y sus componentes (el cociente del área fotosintética y la tasa de asimilación neta) se calcularon de acuerdo a Cardillo y Bernal (2006): el morfológico (CAF, cociente de área fotosintética, en $\mathrm{cm}^{2} \mathrm{~g}^{-1}$ ) es el total del área fotosintética por unidad de biomasa total, y el componente fisiológico (TAN, tasa de asimilación neta, en $\mathrm{mg} \mathrm{día}^{-1} \mathrm{~cm}^{-2}$ ) que mide la asignación de biomasa resultado de la diferencia entre la tasa fotosintética neta diaria y la respiración (Evans, 1972).

$$
T R C=\frac{\left(B T_{2}-B T_{1}\right)}{T_{2}-T_{1}} \times \frac{2}{\left(B T_{1}+B T_{2}\right)}, \quad T A N=\frac{\left(B T_{2}-B T_{1}\right)}{T_{2}-T_{1}} \times \frac{2}{\left(A F T_{1}+A F T_{2}\right)}
$$

Donde: $\mathrm{BT}_{1} \mathrm{y} \mathrm{BT}_{2}$ son la biomasa promedio de las plantas en las cosechas 1 y $2 ; \mathrm{T}_{1}$ y $_{2}$ son el tiempo inicial y final de las dos cosechas; $\mathrm{AFT}_{1} \mathrm{y} \mathrm{AFT}_{2}$, son el total del área fotosintética en la primera y segunda cosecha. La TRC también se puede obtener mediante el producto de CAF $\times$ TAN (Cardillo \& Bernal, 2006).

La proporción raíz: vástago se obtuvo del cociente de la biomasa de las raíces entre la biomasa de los vástagos y no tiene unidades, los valores mayores a la unidad indican que las plántulas asignan más biomasa a la raíz que a la parte aérea (Miquelajauregui \& Valverde, 2010).

Análisis estadísticos: Todos los análisis se llevaron a cabo con el programa SAS (SAS/ Institute, 2002), con un nivel de significancia $(\alpha=0.05)$. El efecto de los tratamientos en la tasa relativa de crecimiento (TRC) y sus componentes (TAN, CAF), así como la altura del tallo y el cociente raíz/vástago se analizaron con ANOVA de tres vías, tomando como factores los niveles de luz, el tipo de suelo y la procedencia de las plántulas de $M$. pugana. Las diferencias entre tratamientos se analizaron con la prueba de comparación múltiple de Tukey's $(\alpha=0.05)$. Las variables que no cumplieron los supuestos para aplicar el análisis en ANOVA fueron transformadas. Las diferencias entre tratamientos en el número de hojas fueron analizadas con la prueba no paramétrica Kruskal-Wallis $(\alpha=0.05)$. El grado de asociación entre la TRC y sus componentes se realizó mediante el Coeficiente de correlación de Pearson $(\alpha=0.05)$.

\section{RESULTADOS}

Tasa relativa de crecimiento (TRC, $\mathrm{g} \mathrm{g}^{-1}$ día $^{-1}$ ): El crecimiento fue afectado por el nivel de luz (Cuadro 2), en la luz alta las plántulas crecieron $37 \%$ más que en la luz baja (luz alta: $0.026 \pm 0.002$; luz baja: $0.019 \pm 0.002$ ). La procedencia de las plántulas y la fertilidad del suelo no afectaron el crecimiento. Sin embargo, en la interacción procedencia de las plántulas $\times$ luz, las plántulas de San Nicolás crecieron $70 \%$ más en el tratamiento de luz alta, en comparación con el de la luz baja, mientras que las plántulas del CUCBA no revelaron diferencias (Cuadro 3).

Tasa de asimilación neta (TAN, $\mathrm{g} \mathrm{cm}^{-2}$ día $^{-1}$ ): La TAN mostró diferencias por efecto de los tratamientos de luz (Cuadro 2). Las plántulas aumentaron la TAN $150 \%$ más en luz alta en comparación con las de la luz baja (luz alta: $0.00035 \pm 0.00004$; luz baja: 0.00014 $\pm 0.00001)$. También hubo diferencias en la interacción luz $\times$ procedencia de las plántulas; mientras que, para las plántulas de $\mathrm{SN}$ revelaron diferencias, en el tratamiento de luz alta mostraron $333 \%$ mayor TAN que en luz baja (Cuadro 3). El factor fertilidad del suelo no presentó diferencias en sus niveles ni en sus interacciones. 


\section{CUADRO 2}

Resultados de ANOVA para la tasa relativa de crecimiento (TRC), cociente de área foliar (CAF), tasa de asimilación neta (TAN), cociente raíz/vástago (C: R/V) y altura del tallo

TABLE 2

ANOVA results for relative growth rate, leaf area ratio, net assimilation rate, root-shoot ratio and stem height

\begin{tabular}{|c|c|c|c|c|c|c|c|c|c|c|}
\hline \multirow[b]{2}{*}{ Factor } & \multicolumn{2}{|c|}{ TRC } & \multicolumn{2}{|c|}{$\mathrm{CAF}$} & \multicolumn{2}{|c|}{ TAN } & \multicolumn{2}{|c|}{ Altura del tallo } & \multicolumn{2}{|c|}{$\mathrm{C}: \mathrm{R} / \mathrm{V}$} \\
\hline & $\begin{array}{l}\text { Valor } \\
\text { de } F\end{array}$ & $\begin{array}{l}\text { Valor } \\
\text { de } P\end{array}$ & $\begin{array}{l}\text { Valor } \\
\text { de } F\end{array}$ & $\begin{array}{l}\text { Valor } \\
\text { de } P\end{array}$ & $\begin{array}{l}\text { Valor } \\
\text { de } F\end{array}$ & $\begin{array}{l}\text { Valor } \\
\text { de } P\end{array}$ & $\begin{array}{l}\text { Valor } \\
\text { de } F\end{array}$ & $\begin{array}{l}\text { Valor } \\
\text { de } P\end{array}$ & $\begin{array}{l}\text { Valor } \\
\text { de } F\end{array}$ & $\begin{array}{l}\text { Valor } \\
\text { de } P\end{array}$ \\
\hline Luz & 4.9 & $0.03 *$ & 30.1 & $0.0^{*}$ & 21.3 & $0.0^{*}$ & 3.6 & 0.07 & 28.3 & $0.0^{*}$ \\
\hline Fertilidad del suelo & 1.05 & 0.31 & 0.9 & 0.33 & 0.97 & 0.33 & 14.5 & $0.0^{*}$ & 1.3 & 0.25 \\
\hline Procedencia de las plántulas & 0.87 & 0.35 & 1.6 & 0.98 & 0.0 & 0.98 & 0.3 & 0.58 & 7.2 & $0.0^{*}$ \\
\hline $\mathrm{L} * \mathrm{~F}$ & 0.16 & 0.68 & 0.6 & 0.68 & 0.16 & 0.69 & 6.8 & $0.02 *$ & 9.5 & $0.0^{*}$ \\
\hline $\mathrm{L} * \mathrm{P}$ & 4.6 & $0.03 *$ & 4.4 & $0.031 *$ & 4.7 & $0.03 *$ & 0.74 & 0.4 & 0.2 & 0.67 \\
\hline $\mathrm{F}^{*} \mathrm{P}$ & 0.02 & 0.87 & 0.9 & 0.96 & 0.0 & 0.96 & 0.45 & 0.5 & 0.5 & 0.47 \\
\hline $\mathrm{L} * \mathrm{~F} * \mathrm{P}$ & 0.44 & 0.51 & 1.1 & 0.93 & 0.01 & 0.93 & 0.05 & 0.82 & 0.3 & 0.61 \\
\hline
\end{tabular}

*Valor de significancia $(\mathrm{P}<0.05), \mathrm{L}=\mathrm{Luz}, \mathrm{F}=$ Fertilidad debido a la procedencia del suelo, $\mathrm{P}=$ Procedencia de plántula.

\section{CUADRO 3}

Promedios de la tasa relativa de crecimiento (TRC), tasa de asimilación neta (TAN) y cociente del área foliar (CAF), altura del tallo y cociente: raíz/vástago

TABLE 3

Averages of relative growth rate, net assimilation rate and leaf area ratio, stem height and root-shoot ratio

\begin{tabular}{|c|c|c|c|}
\hline \multicolumn{4}{|c|}{ Tasa relativa de crecimiento $\left(\mathrm{mg}^{-1} \mathrm{~g}^{-1}\right.$ día $\left.^{-1}\right)$} \\
\hline \multicolumn{2}{|c|}{ Luz alta } & \multicolumn{2}{|c|}{ Luz baja } \\
\hline Plántulas de CUCBA & Plántulas de SN & Plántulas de CUCBA & Plántulas de SN \\
\hline $0.0213 \pm 0.003^{\mathrm{ab}}$ & $0.03 \pm 0.003^{\mathrm{a}}$ & $0.021 \pm 0.001^{\mathrm{ab}}$ & $0.0176 \pm 0.003^{\mathbf{b}}$ \\
\hline \multicolumn{4}{|c|}{ Tasa de asimilación neta $\left(\mathrm{cm}^{2} \mathrm{~g}^{-1}\right)$} \\
\hline \multicolumn{2}{|c|}{ Luz alta } & \multicolumn{2}{|c|}{ Luz baja } \\
\hline Plántulas de CUCBA & Plántulas de SN & Plántulas de CUCBA & Plántulas de SN \\
\hline $0.0003 \pm 0.00005^{\mathrm{ab}}$ & $0.0004 \pm 0.00006^{\mathbf{a}}$ & $0.00016 \pm 0.001^{\mathbf{b c}}$ & $0.00012 \pm 0.00002^{\mathbf{c}}$ \\
\hline \multicolumn{4}{|c|}{ Coeficiente del área foliar $\left(\mathrm{cm}^{2} \mathrm{~g}^{-1}\right)$} \\
\hline \multicolumn{2}{|c|}{ Luz alta } & \multicolumn{2}{|c|}{ Luz baja } \\
\hline Plántulas de CUCBA & Plántulas de SN & Plántulas de CUCBA & Plántulas de SN \\
\hline $79 \pm 3.6^{\mathbf{b}}$ & $74 \pm 2.5^{\mathbf{b}}$ & $138 \pm 4.9^{\mathrm{a}}$ & $143 \pm 4.4^{\mathrm{a}}$ \\
\hline \multicolumn{4}{|c|}{ Altura del tallo $(\mathrm{cm})$} \\
\hline \multicolumn{2}{|c|}{ Fertilidad alta (suelo de SN) } & \multicolumn{2}{|c|}{ Fertilidad baja (suelo del CUCBA) } \\
\hline Plántulas del CUCBA & Plántulas de SN & Plántulas de CUCBA & Plántulas de SN \\
\hline $8.5 \pm 0.5^{\mathbf{b c}}$ & $9.1 \pm 0.5^{\mathbf{b c}}$ & $8.2 \pm 0.1^{\mathrm{c}}$ & $11.1 \pm 0.4^{\mathrm{a}}$ \\
\hline \multicolumn{4}{|c|}{ Cociente: raíz/vástago } \\
\hline \multicolumn{2}{|c|}{ Fertilidad alta (suelo de SN) } & \multicolumn{2}{|c|}{ Fertilidad baja(suelo del CUCBA) } \\
\hline Luz alta & Luz baja & Luz alta & Luz baja \\
\hline $1.7 \pm 0.1^{b}$ & $1.5 \pm 0.07^{\mathbf{b c}}$ & $2.2 \pm 0.1^{\mathrm{a}}$ & $1.3 \pm 0.05^{\mathbf{c}}$ \\
\hline
\end{tabular}

Promedios \pm error estándar. Acrónimos: $\mathrm{CUCBA}=$ Centro Universitario de Ciencias Biológicas y Agropecuarias, $\mathrm{SN}=\mathrm{San}$ Nicolás. Letras en negritas significan diferencias entre tratamientos según la prueba de Tukey HSD, valor de significancia $=(\mathrm{P}<0.05)$. 


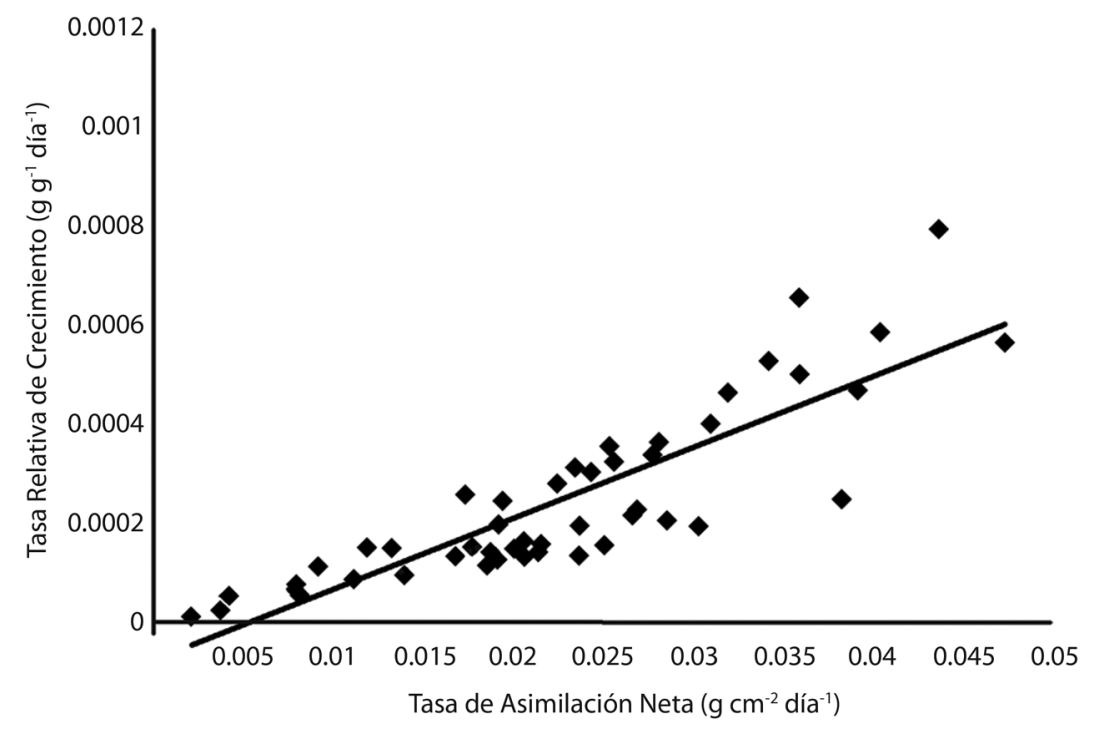

Fig. 1. Asociación entre la tasa relativa de crecimiento y la tasa de asimilación neta.

Fig. 1. Relationship between relative growth rate and net assimilation rate.

Cociente del área foliar (CAF, $\left.\mathrm{cm}^{2} \mathrm{~g}^{-1}\right)$ : La asignación de biomasa a las hojas aumento como respuesta a la disminución de la luz (Cuadro 2), en el tratamiento de luz baja aumentó $83 \%$ en contraste con el de la luz alta (luz alta: $76.7 \pm 2.2$; luz baja: $140.3 \pm 0.002$ ). Además, el efecto de la luz fue distinto por la procedencia de las plántulas, en la luz baja las plántulas de San Nicolás y las del CUCBA aumentaron $93 \%$ y $74 \%$ el CAF, respectivamente en contraste con la luz alta, (Cuadro 3). La fertilidad del suelo no mostró significancia ni sus interacciones.

Altura del tallo (cm) y número de hojas: La altura final del tallo varió por efecto de la procedencia de las plántulas $(F=14.5, \mathrm{P}<$ $0.01)$, las plántulas de $\mathrm{SN}$ fueron $20 \%$ más altas que las del CUCBA (CUCBA $=8.4$ $\pm 0.2, \mathrm{SN}=10.1 \pm 0.4$ ). Los factores luz y fertilidad del suelo no revelaron diferencias significativas. Pero el efecto de la fertilidad fue dependiente de la procedencia de las plántulas (Cuadro 2), en el suelo del CUCBA las plántulas de SN fueron $35 \%$ más altas que las del CUCBA (Cuadro 3). El número de hojas no reveló diferencias significativas por efecto de los tratamientos.

Cociente raíz/vástago (C: $\mathrm{R} / \mathrm{V})$ : La asignación de biomasa a la parte aérea y subterránea en las plántulas varió debido a los niveles de luz $(F=28.9, \mathrm{P}<0.01)$, fue $37 \%$ mayor en el tratamiento de luz alta en comparación con el de luz baja (luz alta: $1.9 \pm 0.1$; luz baja: $1.4 \pm 0.05)$. Los factores procedencia de las plántulas y fertilidad de suelo no presentaron diferencias significativas. Sin embargo, el efecto de la fertilidad del suelo fue dependiente del nivel de luz (Cuadro 2); las plántulas revelaron mayor $\mathrm{C}: \mathrm{R} / \mathrm{V}$ en el suelo del CUCBA con el nivel de luz alto, mientras que el menor fue en la luz baja también en el suelo del CUCBA (Cuadro 3).

Correlación entre la tasa relativa de crecimiento y sus componentes: La TAN se correlacionó positivamente con la TRC $\left(R^{2}=\right.$ $0.74, \mathrm{P}=>0.01$ (Fig. 1). En general la TAN fue la variable que mayormente predijo la variación en la TRC de las plántulas de M. pugana; mientras que el CAF no mostró correlación. 


\section{DISCUSIÓN}

La fase de plántula es especialmente vulnerable a los cambios ambientales (Kitajima, 1994), mientras que la falta de luz es el factor más limitante para su crecimiento (Poorter \& Arets, 2003). En este trabajo las plántulas de Magnolia pugana en general crecieron aproximadamente $40 \%$ más en presencia de luz alta. Sin embargo; este factor sólo afectó significativamente a la población de San Nicolás, donde las plántulas crecieron $58.6 \%$ más en luz alta que en el de luz baja. Estos resultados son similares con los encontrados por SaldañaAcosta, Meave y Sánchez-Velásquez (2009), en plántulas de cinco especies del bosque mesófilo de montaña en la Reserva de la Biósfera de Manantlán en Jalisco, (Citharexylum mocinnii D. Don, Dendropanax arboreus (L.) Decne. et Planch, Fraxinus uhdei Lingelsh, Magnolia iltisiana A. Vázquez, Quercus salicifolia Née; sometidas a diferentes niveles de luz las cuáles mostraron mayor crecimiento en el nivel de luz más alto. A su vez, están de acuerdo con Poorter y Garnier (1999), que sugieren que las plántulas de leñosas en la sombra presentan tasas de crecimiento bajas, hasta la formación de un claro en el dosel del bosque, entonces el aumento de luz conduce a un mayor crecimiento.

La plasticidad en las plantas está asociada a la habilidad competitiva en un ambiente particular (Lambers et al., 1998). Así mismo, Sultan (2000) señala que un solo genotipo puede producir diferentes fenotipos en diferentes entornos. En este trabajo se encontró que las plántulas obtenidas de la población de San Nicolás (silvestre), mostraron mayor plasticidad fenotípica que las de la población del CUCBA (cultivada). Este comportamiento podría deberse a que la población del CUCBA se originó de un lote de semillas procedentes de muy pocos individuos posiblemente emparentados provenientes de una población pequeña y aislada, por lo que puede estar sujeta a una mayor deriva génica y endogamia, en contraste la población de San Nicolás.
Las plántulas que estuvieron sometidas a la luz baja mostraron aproximadamente el doble de área foliar por unidad de biomasa (CAF), en comparación con las que estuvieron en luz alta. Villar et al. (2004) mencionan que las plantas que crecen en ambientes sombreados asignan más biomasa a los órganos fotosintéticos para incrementar el área de exposición y maximizar la intercepción de la luz. Por lo tanto, la asignación de recursos al área foliar es un factor clave en la eficiencia fotosintética para la captación de luz en plántulas que crecen bajo el dosel (Bazzaz \& Wayne, 1994; Kitajima, 1994). Aunque el CAF de las plántulas con distinta procedencia no fue afectado por los niveles de luz, las plántulas de $\mathrm{SN}$ fueron más sensibles a los cambios de luz. En consecuencia, las plántulas de SN están más adaptadas a las condiciones sombra y por lo tanto son más eficientes en la captura de luz (Poorter \& Garnier, 1999).

La tasa de asimilación neta puede ser un buen indicador de la tasa de fotosíntesis por unidad de área foliar (Poorter, 2001). Las plántulas mostraron mayor tasa de asimilación neta (TAN) en presencia de luz alta en contraste con luz baja. Estos resultados están de acuerdo con los encontrados por otros autores (Veneklaas \& Poorter, 1998; Shipley, 2002), que mencionan que las plantas que crecen en niveles de luz altos registran tasas de fijación de carbono mayores. En este estudio a pesar de que la procedencia de las plántulas no mostró diferencias significativas, las de San Nicolás tuvieron 18.5 $\%$ mayor TAN que las del CUCBA. Estudios han documentado que especies con tasa de asimilación alta y mayor asignación de biomasa en los tallos muestran mayor potencial de competitividad intraespecífica por recursos como la luz, que es un factor clave para la regeneración (Poorter \& Arets, 2003).

El crecimiento de las plantas depende de su habilidad para capturar recursos como luz y nutrientes (Tilman, 1985). En este estudio la longitud del tallo varió debido a la procedencia de las plántulas, en el suelo del CUCBA los tallos de las de SN fueron $26 \%$ más altos que los de las del CUCBA. Se ha demostrado que el crecimiento en altura del tallo en especies de 
Magnolia difiere debido a la procedencia de las semillas (Shu, Yang, \& Yang, 2012). Es posible que la variación encontrada entre las dos poblaciones estudiadas (CUCBA y SN) se deba a la mayor plasticidad fenotípica en las plántulas procedentes de SN. Shu et al (2009), sugieren que la altura de la planta podría ser un indicador indirecto para seleccionar plántulas en estudios con especies de diferentes procedencias.

El crecimiento de las plántulas presentó una correlación positiva con el componente fisiológico (TAN), por lo que éste, aporta la mayor contribución en la variación del crecimiento en $M$. pugana. Pompa y Bongers (1988), mencionan que, para las especies tolerantes a la sombra, la eficiencia en la economía del carbono toma gran importancia en ambientes con poca disponibilidad de luz. Además, cuando la disponibilidad de luz se incrementa las plantas maximizan la supervivencia, ganancia de carbono y por lo tanto del crecimiento (Kitajima, 1994).

Las plántulas mostraron mayor crecimiento de la raíz en presencia de luz alta. Canham et al. (1996), mencionan que conforme el nivel de luz incrementa, la disponibilidad de recursos del suelo tiene un mayor efecto sobre el crecimiento de las plántulas. Similares resultados se encontraron en el presente estudio, en el que el suelo influyó en la asignación de biomasa a la raíz al interactuar con el nivel de luz, en el tratamiento de menor fertilidad (CUCBA) con la mayor cantidad de luz. Esto se debe a que una mayor radiación maximiza la tasa fotosintética, lo cual implica una mayor absorción de nutrientes ya que el crecimiento es estimulado, así como un aumento en la tasa de absorción de agua por la transpiración (Poorter \& Nagel, 2000).

Las plantas muestran una amplia variación en su crecimiento, la TRC es utilizada para analizar las causas inherentes al crecimiento (Poorter \& Nagel, 2000). Numerosos estudios (Lambers et al., 1998; Poorter \& Garnier, 1999; Poorter, 2001), han demostrado que la tasa de asimilación neta es dependiente de las intensidades de luz altas; mientras que el cociente del área foliar varía inversamente debido a que es el factor dominante en la luz baja. En este trabajo encontramos que la variación en el crecimiento en las plántulas de $M$. pugana está asociado a la TAN, por lo que disminuyeron su área foliar cuando estuvieron sometidas a la luz alta para mantener su balance de agua con la reducción de la respiración, con lo que podrían evitar la fotoinhibición (Shipley, 2002).

Estos resultados sugieren que las poblaciones de $M$. pugana podrían cambiar sus respuestas fisiológicas y por lo tanto su desempeño, debido a cambios en recursos como la luz y el suelo. Esta alta plasticidad fenotípica es importante para afrontar dichos cambios y podría ser una ventaja para adaptarse a diversos ambientes de competencia en los bosques de galería donde habita la especie.

\section{AGRADECIMIENTOS}

Los autores agradecen a Adriana Avendaño por su apoyo logístico. También a Alejandro Muñoz, Oscar Carvajal, Edgar López y Gil Virgen por su apoyo técnico. Así como a los revisores por sus sugerencias y comentarios que ayudaron a mejorar el escrito.

\section{RESUMEN}

El estudio de la ecofisiología de las especies en peligro de extinción es clave para el éxito de programas de conservación y restauración ecológica. El objetivo de este trabajo fue conocer el efecto de los factores luz, fertilidad de suelo y procedencia de las plántulas en el crecimiento de plántulas de Magnolia pugana. Se estimó la tasa relativa de crecimiento (TRC) y sus componentes (Tasa de Asimilación Neta: TAN y Cociente del Área Foliar: CAF), así como la relación raíz/vástago $(\mathrm{C}: \mathrm{R} / \mathrm{V})$. Las plántulas fueron obtenidas de semillas recolectadas de dos localidades en Zapopan, Jalisco, México, la primera es una población silvestre en San Nicolás (SN) y la segunda es una plantación ubicada en los jardines del Centro Universitario de Ciencias Biológicas y Agropecuarias (CUCBA). El experimento se llevó a cabo en condiciones de invernadero. En septiembre 2015, 96 plántulas de tres meses de edad fueron sometidas a los siguientes tratamientos con un experimento factorial $(2 \times 2 \times 2)$ : nivel de luz (alta $=1120 \mu \mathrm{mol} \mathrm{m}^{-2} \mathrm{~s}^{-1}$ y baja $\left.=136.3 \mu \mathrm{mol} \mathrm{m}^{-2} \mathrm{~s}^{-1}\right)$, procedencia de las plántulas (San Nicolás y CUCBA) y fertilidad del suelo (alta = suelo San Nicolás, baja $=$ suelo del CUCBA) con 12 repeticiones por cada combinación de factores y niveles. El crecimiento 
se estimó con dos cosechas: la primera a los 30 días de establecido el experimento y la segunda a los 60 días. Los niveles de luz, fertilidad del suelo y la procedencia de las plántulas influyeron en el crecimiento a través de ajustes fisiológicos y morfológicos. En general Magnolia pugana mostró mayor TRC y TAN en la luz alta, mientras que el CAF disminuyó. La fertilidad del suelo y la procedencia de las plántulas no afectaron la TRC ni sus componentes. Sin embargo, si fueron afectados por los niveles de luz, las plántulas de SN crecieron más en luz alta. La altura del tallo varió debido a la fertilidad del suelo y a la procedencia de las plántulas, en el suelo con fertilidad baja, en el suelo del CUCBA las plántulas de SN fueron $35 \%$ más altas que las del CUCBA. El suelo de baja fertilidad en la luz alta ocasionó que las plántulas asignaran mayor biomasa a la raíz. El componente fisiológico (TAN) fue el mayor determinante en la variación intraespecífica de la TRC. Las plántulas de $\mathrm{SN}$ mostraron mayor plasticidad fenotípica debido a que es una población silvestre, por lo que posiblemente tiene variación genética más alta que la población cultivada del CUCBA. Los resultados sugieren que Magnolia pugana es una especie con capacidad de adaptarse a diversos ambientes debido a su plasticidad fenotípica frente a los distintos niveles de luz y fertilidad del suelo.

Palabras clave: Magnolia pugana; tasa relativa de crecimiento; cociente de área foliar; tasa de asimilación neta; análisis de crecimiento; especies amenazadas.

\section{REFERENCIAS}

Acevedo-Rosas, R., Hernández-Galaviz, M., \& CházaroBasáñez, M. (2008). Especies de plantas vasculares descritas de las barrancas aledañas a la ciudad de Guadalajara y de Río Blanco, Jalisco, México. Polibotánica, 26, 1-38.

Alameda, D., \& Villar, R. (2009). Moderate soil compaction: implications on growth and architecture in seedlings of 17 woody plant species. Soil and Tillage Research, 103, 325-331.

Azcon-Bieto, J., \& Talon, M. (2008). Fundamentos de fisiología vegetal. Madrid, España: McGraw Hill Interamericana.

Bazzaz, F. A., \& Wayne, P. M. (1994). Coping with environmental heterogeneity: the physiological ecology of tree seedling regeneration across the gapunderstory continuum. In M. M. Caldwell \& R. W. Pearcy (Eds.), Exploitation of environmental heterogeneity by plants: ecophysiological processes above and belowground (pp. 349-390). New York: Academic Press.

Bewley, J. D., \& Black, M. (1994). Seeds. Physiology of development and germination. Nueva York, USA: Plenum Press.
Canham, C. D., Berkowitz, A. R., Kelly, V. R., Lovett, G. M., Ollinger, S. V., \& Schnurr, J. (1996). Biomass allocation and multiple resource limitation in tree seedlings. Canadian Journal of Forest Research, 26(9), 1521-1530.

Cardillo, E., \& Bernal, C. J. (2006). Morphological response and growth of cork oak (Quercus suber L.) seedlings at different shade levels. Forest Ecology and Management, 222, 296-301.

Carranza, A. S. (2014). Diversidad y diferenciación genética de Magnolia pugana y Magnolia pacifica, especies endémicas del occidente de México (Tesis de pregrado). Universidad de Guadalajara, Guadalajara, Jalisco, México.

Cicuzza, D., Newton, A. C., \& Oldfield, S. (2007). Red List of Magnoliaceae. Cambridge, UK: Fauna \& Flora International.

Evans, G. C. (1972). The quantitative analysis of plant growth. California, USA: Blackwell Scientific Publications.

Fenner, M., \& Kitajima, K. (1999). Seed and seedling ecology. In F. I. Pugnaire \& F. Valladares (Eds.), Handbook of functional plant ecology (pp. 589-622). New York: Marcel Dekker.

García-Ramírez, Y., Freire-Seijo, M., Tejeda, M., \& Reyes, M. (2007). Germinación in vitro de semillas de Dendrocalamus strictus (Rosb.) Nees. Biotecnología Vegetal, 7(1), 41-44.

Glimn-Lacy, J., \& Kaufman, P. B. (2006). Botany illustrated: introduction to plants, major groups, flowering plant families. New York, USA: Springer Science.

Herrera, J., Alizaga, R., Guevara, E., \& Jiménez, V. (2006). Germinación y crecimiento de la planta. Fisiología de la producción de los cultivos tropicales. San José, Costa Rica: Universidad de Costa Rica.

Hoffmann, W. A., \& Poorter, H. (2002). Avoiding bias in calculations of relative growth rate. Annals of Botany, $80,37-42$.

Huante, P., Rincon, E., \& Acosta, I. (1995). Nutrient availability and growth rate of 34 woody species from a tropical deciduous forest in Mexico. Functional Ecology, 9(6), 849-858.

Ibarra, C. D., Ruiz, C. J., González, E. R., \& Flores, J. G. (2007). Distribución espacial del contenido de materia orgánica de los suelos de Zapopan, Jalisco. Terra Latinoamericana, 25(2), 187-194.

Jacobo-Pereira, C., Romo-Campos, R., \& Flores, J. (2016). Germinación de semillas de Magnolia pugana (Magnoliaceae), especie endémica y en peligro de extinción del occidente de México. Botanical Science, 94(3), 1-10. 
Jandel Scientific. (1993). Sigma Scan/Image. Measurement Software for Windows. Jandel Scientific, San Rafael.

Kitajima, K. (1994). Relative importance of photosynthetic traits and allocation patternsas correlates of seedling shade tolerance of 13 tropical trees. Oecologia, 98, 419-428.

Lambers, H., Chapin, III F. S., \& Pons, T. L. (1998). Plant physiological ecology. NewYork, USA: Springer Verlag.

Miquelajauregui, Y., \& Valverde, T. (2010). Survival and early growth of two congeneric cacti that differ in their level of rarity. Journal of Arid Environments, $74,1624-1631$.

Pearcy, R. W., Valladares, F., Wright, S. J., \& de Paulis, E. L. (2004). A functional analysis of the crown architecture of tropical forest Psychotria species: do species vary in light capture efficiency and consequently in carbon gain and growth? Oecologia, 139(2), 163-177.

Poorter, L. (2001). Light-dependent changes in biomass allocation and their importance for growth of rain forest tree species. Functional Ecology, 15(1), 113-123.

Poorter, L., \& Arets, E. J. M. M. (2003). Light environmental and tree strategies in a Bolivian tropical moist forest: an evaluation of the light partitioning hypothesis. Plant Ecology, 166, 295-306.

Poorter, H., \& Garnier, E. (1999). Ecological significance of inherent variation in relative growth rate and its components. In F. I. Pugnaire \& F. Valladares (Eds.), Handbook of functional plant ecology (pp. 81-120). New York: Marcel Dekker.

Poorter, H., \& Nagel, O. (2000). The role of biomass allocation in the growth response of plants to different levels of light, $\mathrm{CO}_{2}$, nutrients and water: a quantitative review. Australian Journal of Plant Physiology, 27(6), 595-607.

Pompa, J., \& Bongers, F. (1988). The effect of canopy gaps on growth and morphology of seedlings rain species. Oecologia, 75, 625-632.

Restrepo, M. L. G., \& Cardona, E. P. (2011). Resultados preliminares del crecimiento temprano en vivero y en parcelas de conservación de varias especies de Magnolias propagadas sexual y asexualmente. En M. L. G. Restrepo (Ed.), Avances en la estrategia para la conservación de las especies de la familia Magnoliaceae en jurisdicción de CORANTIOQUIA (Boletín Técnico Biodiversidad No. 6, pp. 81-94). Medellín: Corantioquia.

Rivers, M. C., Martínez-Salas, E., \& Samain, M. S. (2016). The IUCN Red List of Magnoliaceae. DOI: 10.2305/ IUCN.UK.2014-1.RLTS.T194806A2363344.en
Romo-Campos, R., Flores-Flores, J. L., Flores, J., \& Álvarez-Fuentes, G. (2013). Factores abióticos involucrados en la facilitación entre leñosas y suculentas en el Altiplano Mexicano. Botanical Sciences, 91(3), 319-333.

Ruiz, C. J. A., Flores, L. H. E., Regalado, R. J. R., \& Ramírez, O. G. (2012). Estadísticas climáticas normales del Estado de Jalisco (Libro Técnico Núm. 2). Tepatitlán de Morelos, México: INIFAP-CIRPAC-Campo Experimental Centro Altos de Jalisco.

Saldaña, A. A., Zuloaga, A. M., \& Jardel, P. E. (2001). Germinación de Acer skutchii Rehder y Magnolia iltisiana Vázquez en la Reserva de la Biosfera Sierra de Manantlán, Jalisco, México. Foresta Veracruzana, $3(2), 1-8$.

Saldaña-Acosta, A., Meave, J. A., \& Sánchez-Velásquez, L. R. (2009). Seedling biomass allocation and vital rates of cloud forest tree species: responses to light in shade house conditions. Forest Ecology and Management, 258, 1650-1659.

SAS Institute Inc. 2002. SAS/STAT software, version 9. SAS Institute, Inc., Cary.

SEMARNAT (Secretaría de Medio Ambiente y Recursos Naturales). (2000). Programa de manejo área de protección de flora y fauna La Primavera, México. D. F., México: Secretaría de Medio Ambiente y Recursos Naturales, Comisión Nacional de Áreas Naturales Protegidas.

Sharrock, S. (2012). Estrategia mundial para la conservación de las especies vegetales: una guía para la GSPC, metas, objetivos y datos. Richmond, U.K: Botanic Gardens Conservation International.

Shipley, B. (2002). Trade-offs between net assimilation rate and specific leaf area in determining relative growth rate: relationship with daily irradiance. Functional Ecology, 16, 682-689.

Shu, X., Yang, X., \& Yang, Z. (2012). Variation in seed and seedling traits among fifteen Chinese provenances of Magnolia officinalis. Notulae Botanicae Horti Agrobotanici, 40(2), 274-283.

SPP (Secretaría de Planeación y Presupuesto). (1981). Síntesis geográfica del Estado de Jalisco. D.F., México: Coordinación General de los Servicios Nacionales de Estadística, Geografía e Informática, Secretaría de Planeación y Presupuesto.

Sultan, S. E. (2000). Phenotypic plasticity for plant development, function and life history. Trends in Plant Science, 5(9), 537-542.

Tilman, D. (1985). The resource-ratio hypothesis of plant succession. The American Naturalist, 125(6), 827-852. 
Valladares, F., Aranda, I., \& Sánchez-Gómez, D. (2008). La luz como factor ecológico y evolutivo para las plantas y su interacción con el agua. En F. Valladares (Ed.), Ecología del bosque mediterráneo en un mundo cambiante (pp. 335-369). Madrid: Ministerio de Medio Ambiente, EGRAF, S. A.

Vázquez-García, J. A. (1994). Magnolia (Magnoliaceae) in México and Central America: a synopsis. Brittonia, 46(1), 1-23.

Vázquez-García, J. A., Carbajal, S., \& Hernández, L. L. (2002). Magnolia pugana (Magnoliaceae): una nueva combinación en el complejo Magnolia pacifica. Novon, 12(1), 137-141.

Vázquez-García, J. A., Neill, D. A., Pérez-C., A. J., Arroyo, F., Núñez-V., M. P., Serna, M., ... \& Sánchez, G. A. (2014). Magnoliaceae en el Neotrópico: riqueza, endemismo y estado de conservación. XI Congreso Latinoamericano de Botánica. San Salvador Bahía, Brasil.

Veneklaas, E. J., \& Poorter L. (1998). Growth and carbon partitioning of tropical tree seedlings in contrasting light environments. In H. Lambers, H. Poorter, \& M. M. I. Van Vuuren (Eds.), Inherent variation in plant growth. Physiological mechanisms and ecological consequences (pp. 337-361). Leiden: Backhuys Publishers.

Villar, R., Ruiz-Robleto, J., Quero, J. L., Poorter, H., Valladares, F., \& Marañón, T. (2004). Tasas de crecimiento en especies leñosas: aspectos funcionales e implicaciones ecológicas. En F. Valladares (Ed.), Ecología del bosque mediterráneo en un mundo cambiante (pp. 191-227). Madrid: Ministerio de Medio Ambiente. 